\title{
Design, Synthesis and Inhibitory Properties against Coxsackie B3/B6 of Some Novel Triazole Derivatives
}

\author{
Dongping Shao ${ }^{1}$, Yanbing Yang ${ }^{1}$, Fei Xue ${ }^{1}$, Xianjin Luo ${ }^{1 *}$, Reyila Wubulikasimu ${ }^{1}$, Yuhuan Li $^{2^{*}}$, \\ Rongmei Gao ${ }^{2}$, Weidong Y $\mathrm{e}^{3}$ \\ ${ }^{1}$ School of Chemistry and Chemical Engineering, Shanghai Jiao Tong University, Shanghai, China \\ ${ }^{2}$ Institute of Medicinal Biotechnology, Chinese Academy of Medical Sciences and Peking Union Medical College, \\ Beijing, China \\ ${ }^{3}$ Zhejiang Medicine Co. Ltd., Xinchang Pharmaceutical Factory, \\ Xinchang, China \\ Email: *luoxianjin@sjtu.edu.cn
}

Received January 17, 2013; revised March 12, 2013; accepted April 15, 2013

Copyright (C) 2013 Dongping Shao et al. This is an open access article distributed under the Creative Commons Attribution License, which permits unrestricted use, distribution, and reproduction in any medium, provided the original work is properly cited.

\begin{abstract}
A series of 1,2,4-triazole derivatives were synthesized, and their abilities to inhibit the in vitro replication of Coxsackie B3/B6 were evaluated. Among the 1,2,4-triazole derivatives, compound $3 \mathrm{~g}$ displayed potent activity, with a high antiviral potency $\left(\mathrm{IC}_{50}=1.71 \mu \mathrm{M}\right.$ (against $\mathrm{CVB} 3$ ), $1.43 \mu \mathrm{M}$ (against CVB6)). The structures of all the new synthesized compounds were confirmed by ${ }^{1} \mathrm{H}-\mathrm{NMR}$ spectra, mass spectra and elemental analyses.
\end{abstract}

Keywords: 1,2,4-Triazole; Anti-Enterovirus; Coxsackie B Viruses

\section{Introduction}

In recent years heterocyclic compounds analogues and derivatives have gained significant interest in various fields of drug discovery due to their wide range of biological activities, such as, anti-microbial, anti-tumor, anthelmintic, anti-leishmanial, anti-convulsant and antiinflammatory [1]. And triazoles as important aromatic nitrogen-containing heterocycle possess a lot of desirable features and have been incorporated into a wide variety of potent therapeutic agents [2]. Introduction of a triazole ring into nucleosides to improve bioactivity in antivirus agents has become widespread in drug design practices since the first synthetic nucleoside drug, ribavirin, showed a broad spectrum of antiviral activity against many RNA and DNA viruses.

Human enteroviruses are the most common cause for the majority of human respiratory diseases along with rhinoviruses. Moreover, enteroviruses may cause aseptic meningitis, encephalitis, febrile illness, hand-foot-mouth disease, and myocarditis [3]. It is estimated that worldwide enteroviruses cause 10 - 15 billion (or more) enteroviral infections annually, and many outbreaks have been reported from Asian countries [4]. With more than 200 enterovirus serotypes existing, there is no effective anti-

\footnotetext{
${ }^{*}$ Corresponding authors.
}

viral drug for clinical use of enteroviral infections so far [5]. This highlights the urgency and significance for developing anti-enterovirus agents.

Enteroviruses are non-enveloped, single-stranded (+) RNA viruses belonging to the picornavirus family, which associated with several human and mammalian diseases [6]. Conventionally, enteroviruses were classified into polioviruses, coxsackie A viruses (CVA), coxsackie B viruses (CVB), echoviruses, and enteroviruses $68-71$ (EV 68 - 71). Coxsackie viruses, and in particular CVB 3 , have often been associated with the development of myocarditis, which may lead to sudden death in young adults or progress to dilated cardiomyopathy $[7,8]$.

In our previous work $[9,10]$, a series of 2-pyridyl1H-benzimidazole-4-carboxamide derivatives were synthesized, screened and indentified as modest inhibitors of CVB3. In view of its novel structural template, which differed from those of all reported anti-enterovirus agents, we were interested to introduce 1,2,4-triazoles moiety. A series of amide derivatives containing 1,2,4-triazole ring were synthesized, screened and indentified as modest inhibitors of coxsackie viruses. It was believed that these compounds could be found effective against picornavirus. The inhibitory activities of these triazole derivatives were tested against CVB3, CVB6. No active clinical drugs against enterovirus employed by 
now, a relatively effective drug, ribavirin (RBV), was selected as the positive control drug. These triazole derivatives were found to exhibit good inhibitory activities against the two kinds of enteroviruses.

\section{Experimental}

\subsection{General}

The synthetic procedures for our desired compounds are outlined in Figure 1. Briefly, the triazoles (1) were synthesized with thiosemicarbazide and carboxylic acid [11], and then reacted with halides using sodium hydroxide as base to produce the key intermediates (2) [12]. Only methyl iodide and 4-chloro-1,3-dinitrobenzene could react with the 1,2,4-triazoles in good yield. With another key intermediate 5-methyl-3-phenylisoxazole-4-carboxylic acid (7) in hands, compounds 3 were obtained by one step with excellent yield of $80 \%$ under regular $\mathrm{EDC} /$ HOBt conditions [10]. 3-Chloroperbenzoic acid (MCPBA) was chosen as an oxidant to convert thioethers into sulfones.

The important intermediate 5-methyl-3-phenylisoxazole-4-carboxylic acid (7) was synthesized with benzaldehyde as the starting material (Figure 2). (E)-benzaldehyde oxime (5) was converted into 5-methyl-3-phenylisoxazole-4-carboxylic acid (7) in two steps. A nitrile oxide cycloaddition was employed to generate the trisubstituted isoxazole (6). Then the ester was saponified to yield acid (7). They were used directly without further purification in the next step $[13,14]$.

The virus strains of CVB3, CVB6 were provided by American Type Culture Collection (ATCC). The positive control drug, RBV was produced by Hubei Keyi Pharmaceutical Factory. The newly synthesized triazole compounds (3a-4a) were dissolved in DMSO and diluted with the culture medium. Vero cells were planted in 96-well culture plates. After $24 \mathrm{~h}$ the plates were placed in the corresponding virus bulk for $2 \mathrm{~h}$. Then the solutions of triazole compounds and RBV were added in the plates and cell wells and virus wells were set simultaneously. When the cytopathic effect (CPE) of virus wells was over 4, the CPE of cell wells was observed. The concentration required to inhibit virus growth by $50 \%$ $\left(\mathrm{IC}_{50}\right)$ was determined by the Reed-Muench method [15].

\subsection{Compound Data}

1-(3-(2,4-dinitrophenylthio)-1H-1,2,4-triazol-1-yl)ethano ne (3a).

Yield $77.2 \%$. m.p. $151^{\circ} \mathrm{C} .{ }^{1} \mathrm{HNMR}$ (DMSO, $400 \mathrm{MHz}$ ) $\delta: 2.873\left(\mathrm{~s}, 3 \mathrm{H}, \mathrm{CH}_{3}\right), 7.131-7.154(\mathrm{~d}, 1 \mathrm{H}, \mathrm{ArH}), 8.347$ - 8.376 (m, 1H, ArH), 8.896 - 8.902 (d, 1H, ArH), 8.958 (s, $1 \mathrm{H}, \mathrm{ArH}) . \mathrm{MS} \mathrm{m} / \mathrm{z} 310[\mathrm{M}+\mathrm{H}]^{+}$. Anal. Calcd for $\mathrm{C}_{10} \mathrm{H}_{7} \mathrm{~N}_{5} \mathrm{O}_{5} \mathrm{~S}: \mathrm{C}, 38.84 ; \mathrm{H}, 2.28 ; \mathrm{N}, 22.65 ; \mathrm{S}, 10.37$. Found: C, 38.92; H, 2.07; N, 22.01; S, 11.19 .

(3-(2,4-dinitrophenylthio)-1H-1,2,4-triazol-1-yl)(phen yl)methanone (3b).

Yield $57.2 \%$. m.p. $146^{\circ} \mathrm{C} .{ }^{1} \mathrm{HNMR}$ (DMSO, $400 \mathrm{MHz}$ ) $\delta: 7.378-7.483(\mathrm{~m}, 5 \mathrm{H}, \operatorname{ArH}), 7.510-7.588(\mathrm{~m}, 2 \mathrm{H}$, ArH), 7.694 - $7.715(\mathrm{~d}, 1 \mathrm{H}, \mathrm{ArH}), 7.957-7.978(\mathrm{~d}, 1 \mathrm{H}$, ArH). MS m/z $372[\mathrm{M}+\mathrm{H}]^{+}$. Anal. Calcd for $\mathrm{C}_{15} \mathrm{H}_{9} \mathrm{~N}_{5} \mathrm{O}_{5} \mathrm{~S}$ : C, 48.52; H, 2.44; N, 18.86; S, 8.64. Found: C, 48.83; H, $2.08 ; \mathrm{N}, 18.22 ; \mathrm{S}, 9.32$.

(3-(2,4-dinitrophenylthio)-1H-1,2,4-triazol-1-yl)(5-

$$
\begin{aligned}
& \stackrel{\mathrm{S}}{\mathrm{H}} \mathrm{H}_{2}-\mathrm{C}-\mathrm{N}-\mathrm{NH}_{2}+\mathrm{R}_{1} \mathrm{COOH} \stackrel{\mathrm{a}}{\longrightarrow} \\
& \stackrel{\mathrm{C}}{\longrightarrow}
\end{aligned}
$$

Figure 1. Reagents and conditions: (a) toluene, reflux; (b) Halides, $\mathrm{NaOH}, \mathrm{CH}_{3} \mathrm{CH}_{2} \mathrm{OH}$, rt; (c) $\mathrm{R}_{2} \mathrm{COOH}, \mathrm{EDC} / \mathrm{HOBt}, \mathrm{Et}_{3} \mathrm{~N}$, $\mathrm{CH}_{2} \mathrm{Cl}_{2}, \mathrm{rt}$; (d) $\mathrm{MCPBA}, \mathrm{CH}_{2} \mathrm{Cl}_{2}, 0^{\circ} \mathrm{C} \rightarrow \mathrm{rt}$.

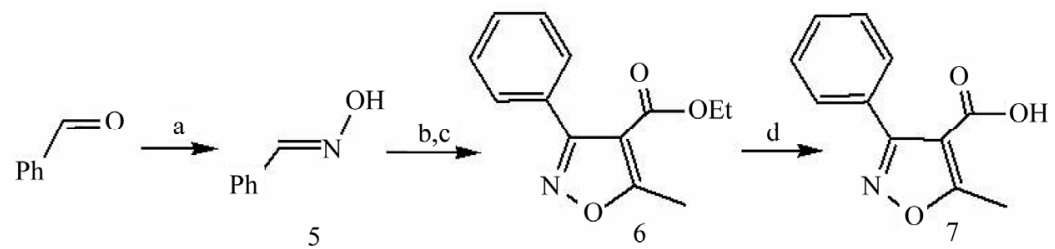

Figure 2. Reagents and conditions: (a) $\mathrm{NH}_{2} \mathrm{OH} \cdot \mathrm{HCl} / \mathrm{Na}_{2} \mathrm{CO}_{3}, \mathrm{CH}_{3} \mathrm{CH}_{2} \mathrm{OH}$, rt; (b) NCS, DMF, rt; (c) $\mathrm{CH}_{3} \mathrm{CH}_{2} \mathrm{ONa} / \mathrm{EAA}$, $\mathrm{CH}_{3} \mathrm{CH}_{2} \mathrm{OH}, 50^{\circ} \mathrm{C}$; (d) $\mathrm{NaOH}, \mathrm{CH}_{3} \mathrm{CH}_{2} \mathrm{OH} / \mathrm{H}_{2} \mathrm{O}$, rt. 
methyl-3-phenylisoxazol-4-yl)methanone (3c).

Yield 66.3\%. m.p. $138^{\circ} \mathrm{C} .{ }^{1} \mathrm{HNMR}$ (DMSO, $400 \mathrm{MHz}$ ) $\delta: 2.673\left(\mathrm{~s}, 3 \mathrm{H}, \mathrm{CH}_{3}\right), 7.378-7.416(\mathrm{~m}, 1 \mathrm{H}, \mathrm{ArH}), 7.448$ - 7.467 (d, 2H, ArH), 7.510 - 7.548 (m, 1H, ArH), 7.569 - $7.588(\mathrm{~m}, 2 \mathrm{H}, \mathrm{ArH}), 7.694$ - 7.715 (d, 1H, ArH), 7.795 - $7.978(\mathrm{~d}, 1 \mathrm{H}, \mathrm{ArH}) . \mathrm{MS} \mathrm{m} / \mathrm{z} 453[\mathrm{M}+\mathrm{H}]^{+}$. Anal. Calcd for $\mathrm{C}_{19} \mathrm{H}_{12} \mathrm{~N}_{6} \mathrm{O}_{6} \mathrm{~S}$ : C, 50.44; $\mathrm{H}, 2.67 ; \mathrm{N}, 18.58 ; \mathrm{S}, 7.09$. Found: C, 50.71; H, 2.83; N, 18.17; S, 8.35.

1-(3-(2,4-dinitrophenylthio)-5-(trifluoromethyl)-1H-1, 2,4-triazol-1-yl)ethanone (3d).

Yield 54.5\%. m.p. $154^{\circ} \mathrm{C} .{ }^{1} \mathrm{HNMR}$ (DMSO, $400 \mathrm{MHz}$ ) $\delta: 2.648\left(\mathrm{~s}, 3 \mathrm{H}, \mathrm{CH}_{3}\right), 7.539-7.587(\mathrm{~m}, 1 \mathrm{H}, \mathrm{ArH}), 8.026$ - 8.044 (m, 1H, ArH), 8.224 (s, 1H, ArH). MS m/z 378 $[\mathrm{M}+\mathrm{H}]^{+}$. Anal. Calcd for $\mathrm{C}_{11} \mathrm{H}_{6} \mathrm{~F}_{3} \mathrm{~N}_{5} \mathrm{O}_{5} \mathrm{~S}: \mathrm{C}, 35.02 ; \mathrm{H}$, $1.60 ; \mathrm{N}, 18.56$; S, 8.50. Found: C, 35.63; H, 2.13; N, $18.22 ; \mathrm{S}, 8.97$.

(3-(2,4-dinitrophenylthio)-5-(trifluoromethyl)-1H-1,2, 4-triazol-1-yl)(phenyl)methanone (3e).

Yield 34.2\%. m.p. $150^{\circ} \mathrm{C}$. ${ }^{1} \mathrm{HNMR}$ (DMSO, $400 \mathrm{MHz}$ ) $\delta$ : $7.482-7.511(\mathrm{~m}, 4 \mathrm{H}, \mathrm{ArH}), 7.571-7.590(\mathrm{~m}, 1 \mathrm{H}$, ArH), 7.654 - 7.675 (m, 1H, ArH), 8.459 - $8.490(\mathrm{~m}, 1 \mathrm{H}$, ArH), $8.745-8.737$ (d, 1H, ArH). MS m/z $440[\mathrm{M}+\mathrm{H}]^{+}$. Anal. Calcd for $\mathrm{C}_{16} \mathrm{H}_{8} \mathrm{~F}_{3} \mathrm{~N}_{5} \mathrm{O}_{5} \mathrm{~S}: \mathrm{C}, 43.74 ; \mathrm{H}, 1.84 ; \mathrm{N}$, 15.94; S, 7.30. Found: C, 44.12; H, 2.04; N, 15.28; S, 8.37 .

(3-(2,4-dinitrophenylthio)-5-(trifluoromethyl)-1H-1,2, 4-triazol-1-yl)(5-methyl-3-phenylisoxazol-4-yl)methanon e (3f).

Yield 54.3\%. m.p. $144^{\circ} \mathrm{C} .{ }^{1} \mathrm{HNMR}$ (DMSO, $400 \mathrm{MHz}$ ) $\delta: 2.673\left(\mathrm{~s}, 3 \mathrm{H}, \mathrm{CH}_{3}\right), 7.378-7.510(\mathrm{~m}, 5 \mathrm{H}, \mathrm{ArH}), 7.571$ - 7.586 (m, 1H, ArH), 7.695 - 7.715 (d, 1H, ArH), 7.797 - $7.978(\mathrm{~d}, 1 \mathrm{H}, \mathrm{ArH}) . \mathrm{MS} \mathrm{m} / \mathrm{z} 521[\mathrm{M}+\mathrm{H}]^{+}$. Anal. Calcd for $\mathrm{C}_{20} \mathrm{H}_{11} \mathrm{~F}_{3} \mathrm{~N}_{6} \mathrm{O}_{6} \mathrm{~S}$ : C, 46.16; H, 2.13; N, 16.15; $\mathrm{S}, 6.16$. Found: C, 46.45; H, 2.01; N, 15.87; S, 7.08.

1-(3-(2,4-dinitrophenylthio)-5-phenyl-1H-1,2,4-triazol -1 -yl)ethanone (3g).

Yield 47.5\% .m.p. $167^{\circ} \mathrm{C} .{ }^{1} \mathrm{HNMR}$ (DMSO, $400 \mathrm{MHz}$ ) $\delta: 2.734$ (s, 3H, $\left.\mathrm{CH}_{3}\right), 7.472-7.557$ (m, 5H, ArH), 8.357 - 8.385 (d, 1H, ArH), 8.552 - 8.581 (m, 1H, ArH), 8.902 - 8.928 (d, 1H, ArH). MS m/z $386[\mathrm{M}+\mathrm{H}]^{+}$. Anal. Calcd for $\mathrm{C}_{16} \mathrm{H}_{11} \mathrm{~N}_{5} \mathrm{O}_{5} \mathrm{~S}: \mathrm{C}, 49.87 ; \mathrm{H}, 2.88 ; \mathrm{N}, 18.17 ; \mathrm{S}, 8.32$. Found: C, 49.98; H, 2.56; N, 17.75; S, 9.04.

(3-(2,4-dinitrophenylthio)-5-phenyl-1H-1,2,4-triazol-1 -yl)(phenyl)methanone (3h).

Yield $46.2 \%$. m.p. $153^{\circ} \mathrm{C}$. ${ }^{1} \mathrm{HNMR}$ (DMSO, $400 \mathrm{MHz}$ ) $\delta: 7.473-7.510(\mathrm{~m}, 4 \mathrm{H}, \mathrm{ArH}), 7.538-7.557(\mathrm{t}, 1 \mathrm{H}$, ArH), $7.771-7.766$ (m, 2H, ArH), $7.881-7.905$ (m, 2H, ArH), $8.276-8.297$ (d, 1H, ArH), $8.356-8.386(\mathrm{~d}, 1 \mathrm{H}$, ArH), $8.552-8.580(\mathrm{~m}, 1 \mathrm{H}, \mathrm{ArH}), 8.904-8.928(\mathrm{~d}, 1 \mathrm{H}$, ArH). MS m/z $448[\mathrm{M}+\mathrm{H}]^{+}$. Anal. Calcd for $\mathrm{C}_{21} \mathrm{H}_{13} \mathrm{~N}_{5} \mathrm{O}_{5} \mathrm{~S}$ : C, 56.37; H, 2.93; N, 15.65; S, 7.17. Found: C, 56.86; H, $2.53 ; \mathrm{N}, 14.83 ; \mathrm{S}, 7.63$.

(3-(2,4-dinitrophenylthio)-5-phenyl-1H-1,2,4-triazol-1yl)(5-methyl-3-phenylisoxazol-4-yl)methanone (3i).
Yield $31.3 \%$. m.p. $145^{\circ} \mathrm{C} .{ }^{1} \mathrm{HNMR}$ (DMSO, $400 \mathrm{MHz}$ ) $\delta: 2.718\left(\mathrm{~s}, 3 \mathrm{H}, \mathrm{CH}_{3}\right), 7.472-7.510(\mathrm{~m}, 5 \mathrm{H}, \mathrm{ArH}), 7.535$ - 7.557 (m, 1H, ArH), 7.721 - 7.766 (m, 2H, ArH), 7.881 - 7.905 (m, 2H, ArH), 8.276 - 8.297 (d, 1H, ArH), 8.356 - 8.385 (q, 1H, ArH), $8.553-8.580$ (q, 1H, ArH). MS $\mathrm{m} / \mathrm{z} 529[\mathrm{M}+\mathrm{H}]^{+}$. Anal. Calcd for $\mathrm{C}_{25} \mathrm{H}_{16} \mathrm{~N}_{6} \mathrm{O}_{6} \mathrm{~S}: \mathrm{C}$, 56.82; H, 3.05; N, 15.90; S, 6.07. Found: C, 57.02; H, $3.21 ; \mathrm{N}, 15.68 ; \mathrm{S}, 6.64$.

1-(3-(methylthio)-1H-1,2,4-triazol-1-yl)ethanone (3j).

Yield $34.5 \%$. m.p. $175^{\circ} \mathrm{C} .{ }^{1} \mathrm{HNMR}$ (DMSO, $400 \mathrm{MHz}$ ) $\delta: 2.718\left(\mathrm{~s}, 3 \mathrm{H}, \mathrm{CH}_{3}\right), 2.734\left(\mathrm{~s}, 3 \mathrm{H}, \mathrm{CH}_{3}\right), 8.904-8.922$ (m, 1H, ArH). MS m/z $158[\mathrm{M}+\mathrm{H}]^{+}$. Anal. Calcd for $\mathrm{C}_{5} \mathrm{H}_{7} \mathrm{~N}_{3} \mathrm{OS}$ : C, 38.20; H, 4.49; N, 26.73; S, 20.40. Found: C, 38.33; H, 4.05; N, 25.95; S, 21.38.

(3-(methylthio)-1H-1,2,4-triazol-1-yl)(phenyl)methano ne (3k).

Yield $36.2 \%$. m.p. $169^{\circ} \mathrm{C} .{ }^{1} \mathrm{HNMR}$ (DMSO, $400 \mathrm{MHz}$ ) $\delta: 2.734\left(\mathrm{~s}, 3 \mathrm{H}, \mathrm{CH}_{3}\right), 7.473-7.557$ (m, 5H, ArH), 8.911 - $8.928(\mathrm{~m}, 1 \mathrm{H}, \mathrm{ArH})$. MS m/z $220[\mathrm{M}+\mathrm{H}]^{+}$. Anal. Calcd for $\mathrm{C}_{10} \mathrm{H}_{9} \mathrm{~N}_{3}$ OS: C, 54.78; H, 4.14; N, 19.16; S, 14.62. Found: C, 55.14; H, 4.01; N, 18.75; S, 15.38 .

(5-methyl-3-phenylisoxazol-4-yl)(3-(methylthio)-1H-1, 2,4-triazol-1-yl)methanone (3I).

Yield 56.1\%. m.p. $165^{\circ} \mathrm{C} .{ }^{1} \mathrm{HNMR}$ (DMSO, $400 \mathrm{MHz}$ ) $\delta: 2.718\left(\mathrm{~s}, 3 \mathrm{H}, \mathrm{CH}_{3}\right), 2.734\left(\mathrm{~s}, 3 \mathrm{H}, \mathrm{CH}_{3}\right), 7.473-7.510$ (m, 5H, ArH), $8.904-8.922$ (m, 1H, ArH). MS m/z 301 $[\mathrm{M}+\mathrm{H}]^{+}$. Anal. Calcd for $\mathrm{C}_{14} \mathrm{H}_{12} \mathrm{~N}_{4} \mathrm{O}_{2} \mathrm{~S}: \mathrm{C}, 55.99 ; \mathrm{H}$, 4.03 ; N, 18.65; S, 10.68. Found: C, 56.33; H, 4.35; N, $18.29 ; \mathrm{S}, 11.32$.

(3-(2,4-dinitrophenylsulfonyl)-1H-1,2,4-triazol-1-yl)(5methyl-3-phenylisoxazol-4-yl)methanone (4a).

Yield $25.1 \%$. m.p. $159^{\circ} \mathrm{C} .{ }^{1} \mathrm{HNMR}$ (DMSO, $400 \mathrm{MHz}$ ) $\delta: 2.673\left(\mathrm{~s}, 3 \mathrm{H}, \mathrm{CH}_{3}\right), 7.378-7.548(\mathrm{~m}, 5 \mathrm{H}, \mathrm{ArH}), 7.564$ - $7.588(\mathrm{~m}, 2 \mathrm{H}, \mathrm{ArH}), 7.694-7.715$ (d, 1H, ArH). MS $\mathrm{m} / \mathrm{z} 485[\mathrm{M}+\mathrm{H}]^{+}$. Anal. Calcd for $\mathrm{C}_{19} \mathrm{H}_{12} \mathrm{~N}_{6} \mathrm{O}_{8} \mathrm{~S}: \mathrm{C}$, 47.11; H, 2.50; N, 17.35; S, 6.62. Found: C, 47.43; H, $2.32 ; \mathrm{N}, 17.08 ; \mathrm{S}, 7.57$.

\section{Results and Discussion}

The cytotoxicity and antiviral potency of these synthesized 1,2,4-triazole derivatives were evaluated in vero cells against $\mathrm{CVB} 3 / \mathrm{B} 6$. No active clinical drugs against CVB3/B6 employed by now, RBV was the recommended clinical antiviral drug in China, the $\mathrm{IC}_{50}$ values of RVB were provided as comparable data.

The results were summarized in Table 1. The antienterovirus activity of each compound was expressed as the concentration of compound that achieved $50 \%$ inhibition $\left(\mathrm{IC}_{50}\right)$ of enterovirus growth. The cytotoxicity of each compound was expressed as the concentration of compound required to kill $50 \%\left(\mathrm{TC}_{50}\right)$ of the vero cells. As a major pharmaceutical parameter for possible future clinical development, the selectivity index (SI) was de- 
Table 1. Activity of the triazole derivatives against coxsackie virus B3/B6 in vero cells.

\begin{tabular}{|c|c|c|c|c|c|c|c|c|c|}
\hline \multirow{2}{*}{ Compound } & \multirow{2}{*}{$\mathrm{R}_{1}$} & \multirow{2}{*}{$\mathrm{R}_{2}$} & \multirow{2}{*}{$\mathrm{R}_{3}$} & \multicolumn{3}{|c|}{ CVB3 } & \multicolumn{3}{|c|}{ CVB6 } \\
\hline & & & & $\mathrm{TC}_{50}{ }^{\mathrm{a}}(\mu \mathrm{M})$ & $\mathrm{IC}_{50}{ }^{\mathrm{b}}(\mu \mathrm{M})$ & $\mathrm{SI}^{\mathrm{c}}$ & $\mathrm{TC}_{50}^{\mathrm{a}}(\mu \mathrm{M})$ & $\mathrm{IC}_{50}{ }^{\mathrm{b}}(\mu \mathrm{M})$ & $\mathrm{SI}^{\mathrm{c}}$ \\
\hline $3 a$ & $\mathrm{H}$ & & $\mathrm{CH}_{3}$ & 7.41 & 2.47 & 3.0 & 7.41 & 1.43 & 5.2 \\
\hline $3 b$ & $\mathrm{H}$ & & $\mathrm{Ph}$ & 7.41 & 2.47 & 3.0 & 7.41 & $>2.47$ & -- \\
\hline $3 c$ & $\mathrm{H}$ & & & 96.15 & 22.22 & 4.3 & 96.15 & $>22.22$ & -- \\
\hline $3 d$ & $\mathrm{CF}_{3}$ & & $\mathrm{CH}_{3}$ & 66.67 & 22.22 & 3.0 & 66.67 & $>22.22$ & -- \\
\hline $3 e$ & $\mathrm{CF}_{3}$ & & $\mathrm{Ph}$ & 138.67 & 38.49 & 3.6 & 138.67 & $>66.67$ & -- \\
\hline $3 \mathrm{f}$ & $\mathrm{CF}_{3}$ & & & 46.22 & 22.22 & 2.1 & 46.22 & $>22.22$ & -- \\
\hline $3 g$ & $\mathrm{Ph}$ & & $\mathrm{CH}_{3}$ & 4.14 & 1.71 & 3.0 & 5.14 & 1.43 & 3.6 \\
\hline $3 \mathrm{~h}$ & $\mathrm{Ph}$ & & $\mathrm{Ph}$ & 138.67 & 66.67 & 2.1 & 138.67 & 28.64 & 4.8 \\
\hline $3 \mathrm{i}$ & $\mathrm{Ph}$ & & & 96.15 & $\mathrm{NA}^{\mathrm{d}}$ & -- & $>200$ & $>66.67$ & -- \\
\hline $3 \mathrm{j}$ & $\mathrm{H}$ & $\mathrm{CH}_{3}$ & $\mathrm{CH}_{3}$ & $>200$ & $>66.67$ & -- & $>200$ & $>66.67$ & -- \\
\hline $3 \mathrm{k}$ & $\mathrm{H}$ & $\mathrm{CH}_{3}$ & $\mathrm{Ph}$ & $>200$ & $>66.67$ & -- & $>200$ & $>66.67$ & -- \\
\hline 31 & $\mathrm{H}$ & $\mathrm{CH}_{3}$ & & $>200$ & $>66.67$ & -- & $>200$ & 66.67 & $>3.0$ \\
\hline $4 a$ & $\mathrm{H}$ & & & 46.22 & NA & -- & 46.22 & $\mathrm{NA}^{\mathrm{d}}$ & -- \\
\hline RVB & - & - & - & 2000 & 384.90 & 5.2 & 2000 & 384.90 & 5.2 \\
\hline
\end{tabular}

${ }^{\mathrm{a}}$ Cytotoxic concentration required to inhibit vero cell growth by $50 \%$; ${ }^{\mathrm{b}}$ Concentration required to inhibit $\mathrm{CVB} 3$ growth by $50 \%$; ${ }^{\mathrm{c}} \mathrm{Selectivity}$ index values equaled to $\mathrm{TC}_{50} / \mathrm{IC}_{50}$; ${ }^{\mathrm{d}} \mathrm{Not}$ active in the largest concentration which were not toxic to vero cells.

termined as the ratio of $\mathrm{TC}_{50}$ to $\mathrm{IC}_{50}$. The bioactivity of each compound was evaluated by the combination of its $\mathrm{IC}_{50}$ and SI.

As shown in Table 1, the 1,2,4-triazole derivatives (3a-4a) were evaluated against CVB3. It is found that most of the synthesized compounds had good antiviral activity, far more active than RVB with $\mathrm{IC}_{50}$ value of $384.90 \mu \mathrm{M}$. Each of the bioactive compounds possessed an $\mathrm{IC}_{50}$ value within the range from $1.71 \mu \mathrm{M}$ to $66.67 \mu \mathrm{M}$, and a $\mathrm{TC}_{50}$ value ranging from $4.14 \mu \mathrm{M}$ to more than 200 $\mu \mathrm{M}$. Analyzing the activities of the synthesized compounds, the following structure-activity relationship (SAR) was observed.

In series 3a-l, $\mathrm{IC}_{50} \mathrm{~s}$ of compounds $\mathbf{3 a}$ and $\mathbf{3 b}\left(\mathrm{IC}_{50}=\right.$ 2.47 and $2.47 \mu \mathrm{M}$, respectively) were better than compounds $\mathbf{3 d}$ and $\mathbf{3 e}\left(\mathrm{IC}_{50}=22.22\right.$ and $38.49 \mu \mathrm{M}$, respec- tively). However, compounds $\mathbf{3 a}$ and $\mathbf{3 b}$ had pronounced cytotoxicity $\left(\mathrm{TC}_{50}=7.41\right.$ and $7.41 \mu \mathrm{M}$, respectively) resulting in similar selective indices of compounds $\mathbf{3 d}$ and 3e. It was indicated that the antiviral activity could be reduced and the cytotoxicity could be enhanced due to the introduction of trifluoromethyl in the $\mathrm{R}_{1}$ position. Compound 3g with phenyl at the $\mathrm{R}_{1}$ position showed higher $\mathrm{IC}_{50}\left(\mathrm{IC}_{50}=1.71 \mu \mathrm{M}\right)$ than compound $\mathbf{3 a}\left(\mathrm{IC}_{50}=\right.$ $2.47 \mu \mathrm{M})$, but it appeared to be more toxic $\left(\mathrm{TC}_{50}=4.14\right.$ $\mu \mathrm{M})$ than compound $\mathbf{3 a}\left(\mathrm{TC}_{50}=7.41 \mu \mathrm{M}\right)$. Compound $\mathbf{3 h}$ with phenyl at the $\mathrm{R}_{1}$ position showed much lower $\mathrm{IC}_{50}$ $\left(\mathrm{IC}_{50}=66.67 \mu \mathrm{M}\right)$ than compound $\mathbf{3 b}\left(\mathrm{IC}_{50}=2.47 \mu \mathrm{M}\right)$, but it appeared to be less toxic $\left(\mathrm{TC}_{50}=138.67 \mu \mathrm{M}\right)$ than compound $\mathbf{3 b}\left(\mathrm{TC}_{50}=7.41 \mu \mathrm{M}\right)$. Generally, the antiviral regularity of this series was inconspicuous.

Compared with compound $\mathbf{3 g}\left(\mathrm{IC}_{50}=1.71 \mu \mathrm{M}\right)$, all 
other derivatives showed equivalent or decreased antiviral activities. Due to the introduction of dinitro phenyl in the $\mathrm{R}_{2}$ position, compounds $\mathbf{3 a - h}$ exhibited good antiviral potency against CVB3.The introduction of methyl in the $\mathrm{R}_{2}$ position resulted in approximately no biological activities, but their cytotoxicities were significantly decreased with $\mathrm{TC}_{50}$ values of more than $200 \mu \mathrm{M}$. Compounds $3 \mathbf{j}-\mathbf{k}\left(\mathrm{IC}_{50}>66.67 \mu \mathrm{M}\right)$ with methyl substituent at the $\mathrm{R}_{2}$ position caused significantly decrease in antiviral activity, but showed lower cytotoxicity with $\mathrm{TC}_{50}$ values of more than $200 \mu \mathrm{M}$.

Compound $3 \mathbf{c}\left(\mathrm{IC}_{50}=22.22 \mu \mathrm{M}\right)$ with moiety of isoxazole at the $\mathrm{R}_{3}$ position displayed lower activity than compounds $\mathbf{3 a}$ and $\mathbf{3 b}\left(\mathrm{IC}_{50}=2.47\right.$ and $2.47 \mu \mathrm{M}$, respectively), but was less cytotoxic with $\mathrm{TC}_{50}$ value of 96.15 $\mu \mathrm{M}$. Compound 3d with methyl at the $\mathrm{R}_{3}$ position showed higher $\mathrm{IC}_{50}\left(\mathrm{IC}_{50}=22.22 \mu \mathrm{M}\right)$ than compound $\mathbf{3 e}$ with phenyl at the $\mathrm{R}_{3}$ position $\left(\mathrm{IC}_{50}=38.49 \mu \mathrm{M}\right)$. Compound $4 \mathbf{a}$ proved inactive against CVB3/B6 in vero cells which was the oxidation product of compound $\mathbf{3 a}$, but the cytotoxicity to vero cells was decreased.

These structure-activity relationship analyses indicated that the introduction of dinitro phenyl in the $\mathrm{R}_{2}$ position was critical for the high antiviral activity, such as $\mathbf{3 a}, \mathbf{3 b}$, 3g. The bioactive compounds $\mathbf{3 a}, \mathbf{3 b}, \mathbf{3 g}$ expressed better $\mathrm{IC}_{50} \mathrm{~S}\left(\mathrm{IC}_{50}=2.47,2.47,1.71 \mu \mathrm{M}\right)$. They could be considered as promising candidates for the development of new derivatives with anti-enterovirus activity and for additional studies concerning the antiviral activity of this group of compounds. The other bioactive compounds $\mathbf{3 c}$ f, 3h showed higher $\mathrm{IC}_{50} \mathrm{~S}\left(\mathrm{IC}_{50}>20 \mu \mathrm{M}\right)$, but they appeared to be less toxic $\left(\mathrm{TC}_{50}=96.15,66.67,138.67\right.$, 46.22, $138.67 \mu \mathrm{M}$, respectively).

Compounds 3a-4a were screened against CVB6 (Table 1). There were only four compounds $\mathbf{3 a}, \mathbf{3 g}, \mathbf{3 h}, \mathbf{3 l}$ shown good antiviral potency against CVB6 $\left(\mathrm{IC}_{50}=1.43\right.$, $1.43,28.64$ and $22.22 \mu \mathrm{M}$, respectively). The most selective compound against CVB6 was compound 3a, with SI of $5.2, \mathrm{IC}_{50}$ of $1.43 \mu \mathrm{M}, \mathrm{TC}_{50}$ of $7.41 \mu \mathrm{M}$. Comparing these results with those shown in Table 1, it was found that the similarity existed among antiviral activities against CVB3,CVB6 of certain compounds (3a, 3g, 3h), indicating that a compound seemed to be effective against other virus if it was effective against one virus of the same family. Compound $\mathbf{3 a}\left(\mathrm{IC}_{50}=2.47\right.$ and $1.43 \mu \mathrm{M}$, respectively) and $3 \mathrm{~g}\left(\mathrm{IC}_{50}=1.71\right.$ and $1.43 \mu \mathrm{M}$, respectively) exhibited good antiviral activities against both CVB3 and CVB6, but performed toxic $\left(\mathrm{TC}_{50}<10 \mu \mathrm{M}\right)$. Compound $\mathbf{3 h}$ displayed lower cytotoxicity to vero cells $\left(\mathrm{TC}_{50}>100 \mu \mathrm{M}\right)$.

\section{Conclusion}

In summary, a novel series of 1,2,4-triazole derivatives (3a-4a) have been synthesized and evaluated for their antiviral activities against CVB3, CVB6 replication in cell culture. Most of the 1,2,4-triazole derivatives pronounced good anti-CVB3 activities and some pronounced good anti-CVB6 activities. Moreover, some of the synthesized compounds had excellent antiviral activity, but they were cytotoxic to cells, such as compounds 3a, 3b and 3g $\left(\mathrm{IC}_{50}<3 \mu \mathrm{M}, \mathrm{TC}_{50}<10 \mu \mathrm{M}\right)$. And some of the synthesized compounds had low cytotoxicities, such as $\mathbf{3 h}\left(\mathrm{IC}_{50}>20 \mu \mathrm{M}, \mathrm{TC}_{50}>100 \mu \mathrm{M}\right)$, but their antiviral activites were not eminent. Further studies are in progress in our laboratories to increase the antiviral potency and decrease cytotoxicity, most importantly, to improve the $\mathrm{TC}_{50} / \mathrm{IC}_{50}$ ratio. The modifications on 1,2,4triazoles moiety display valuable biological activities, and these modifications can be utilized as potent therapeutic agents in future.

\section{Acknowledgements}

We thank Ms. Chen Yan for ${ }^{1}$ HNMR support.

\section{REFERENCES}

[1] A. K. Wahi and A. Singh, "Triazole: Recent Development and Biological Activities," Asian Journal of Biochemical and Pharmaceutical Research, Vol. 2, No. 1, 2011, pp. 193-205.

[2] I. Pibiri and S. Buscemi, "A Recent Portrait of Bioactive Triazoles," Current Bioactive Compounds, Vol. 6, No. 4, 2010, pp. 208-242. doi:10.2174/157340710793237281

[3] J.-H. Chern and K.-S. Shia, "Design, Synthesis, and Structure-Activity Relationships of Pyrazolo[3,4-d]Pyrimidines: A Novel Class of Potent Enterovirus Inhibitors," Bioorganic \& Medicinal Chemistry Letters, Vol. 14, No. 10, 2004, pp. 2519-2525. doi:10.1016/j.bmcl.2004.02.092

[4] H. A. Rotbart, "Treatment of Picornavirus Infections," Antiviral Research, Vol. 53, No. 2, 2002, pp. 83-98. doi:10.1016/S0166-3542(01)00206-6

[5] V. A. Makarov, O. B. Riabova, et al., "Anti-Coxsackievirus B3 Activity of 2-AMINO-3-Nitropyrazolo[1,5-a]Pyrimidines and Their Analogs," Bioorganic \& Medicinal Chemistry Letters, Vol. 15, No. 1, 2005, pp. 37-39. doi:10.1016/j.bmcl.2004.10.043

[6] G. Pürstinger, "Synthesis and Anti-CVB 3 Evaluation of Substituted 5-Nitro-2-Phenoxybenzonitriles," Bioorganic \& Medicinal Chemistry Letters, Vol. 18, No. 18, 2008, pp. 5123-5125. doi:10.1016/j.bmcl.2008.07.099

[7] M. Esfandiarei and B. M. McManus, "Molecular Biology and Pathogenesis of Viral Myocarditis," Annual Review of Pathology: Mechanisms of Disease, Vol. 3, 2008, pp. 127-155.

doi:10.1146/annurev.pathmechdis.3.121806.151534

[8] N. M. Chapman and K. S. Kim, "Persistent Coxsackievirus Infection: Enterovirus Persistence in Chronic Myocarditis and Dilated Cardiomyopathy," Current Topics in Microbiology and Immunology, Vol. 323, 2008, pp. 275292. doi: $10.1007 / 978-3-540-75546-3 \_13$ 
[9] J. Cheng, J. Xie and X. Luo, "Synthesis and Antiviral Activity against Coxsackie Virus B3 of Some Novel Benzimidazole Derivatives," Bioorganic \& Medicinal Chemistry Letters, Vol. 15, No. 2, 2005, pp. 267-269. doi:10.1016/j.bmcl.2004.10.087

[10] F. Xue and X. Luo, "Inhibitory Properties of 2-Substituent1H-Benzimidazole-4-Carboxamide Derivatives against Enteroviruses," Bioorganic \& Medicinal Chemistry, Vol. 19, No. 8, 2011, pp. 2641-2649. doi:10.1016/j.bmc.2011.03.007

[11] D. R. Guda, T. J. Wang and H. M. Cho, "Trimethylsilyl Isothiocyanate (TMSNCS): An Efficient Reagent for the One-Pot Synthesis of Mercapto-1,2,4-Triazoles," Tetrahedron Letters, Vol. 53, No. 39, 2012, pp. 5238-5242. doi:10.1016/j.tetlet.2012.07.054

[12] Y. Dürüst, "Synthesis of Some Novel 1,3,4- and 1,2,4-
Thiadiazole Derivatives," Phosphorus, Sulfur, and Silicon, Vol. 184, No. 11, 2009, pp. 2923-2935. doi:10.1080/10426500802625453

[13] Z. L. Xin and H. Y. Zhao, "Synthesis and Structure-Activity Relationships of Isoxazole Carboxamides as Growth Hormone Secretagogue Receptor Antagonists," Bioorganic \& Medicinal Chemistry Letters, Vol. 15, No. 4, 2005, pp. 1201-1204. doi:10.1016/j.bmcl.2004.11.075

[14] J. H. Kalin and H. K. Zhang, "Chiral Mercaptoacetamides Display Enantioselective Inhibition of Histone Deacetylase 6 and Exhibit Neuroprotection in Cortical Neuron Models of Oxidative Stress," ChemMedChem, Vol. 7, No. 3, 2012, pp. 425-439.

[15] L. J. Reed and H. Muench, "A Simple Method for Estimating Fifty Percent Endpoints," American Journal of Hygiene, Vol. 27, No. 3, 1938, pp. 493-497. 